\title{
Robot-assisted image-guided transcranial magnetic stimulation for somatotopic mapping of the motor cortex: a clinical pilot study
}

\author{
Sven Rainer Kantelhardt • Tommaso Fadini • Markus Finke • Kai Kallenberg • \\ Jakob Siemerkus • Volker Bockermann • Lars Matthaeus • Walter Paulus • \\ Achim Schweikard • Veit Rohde • Alf Giese
}

Received: 14 September 2009/Accepted: 3 November 2009/Published online: 27 November 2009

(C) The Author(s) 2009. This article is published with open access at Springerlink.com

\begin{abstract}
Purpose Shape and exact location of motor cortical areas varies among individuals. The exact knowledge of these locations is crucial for planning of neurosurgical procedures. In this study, we have used robot-assisted imageguided transcranial magnetic stimulation (Ri-TMS) to elicit MEP response recorded for individual muscles and reconstruct functional motor maps of the primary motor cortex. Methods One healthy volunteer and five patients with intracranial tumors neighboring the precentral gyrus were
\end{abstract}

S. R. Kantelhardt $(\bowtie) \cdot$ V. Bockermann $\cdot$ V. Rohde $\cdot$

A. Giese $(\bowtie)$

Department of Neurosurgery,

Georg-August University of Göttingen,

Robert-Koch-Strasse 40,

37075 Göttingen, Germany

e-mail: sven.kantelhardt@med.uni-goettingen.de

e-mail: alf.giese@med.uni-goettingen.de

T. Fadini $\cdot$ W. Paulus

Department of Clinical Neurophysiology,

Georg-August University of Göttingen,

Göttingen, Germany

K. Kallenberg

Department of Neuroradiology,

Georg-August University of Göttingen,

Göttingen, Germany

M. Finke $\cdot$ L. Matthaeus $\cdot$ A. Schweikard

Institute for Robotics und Cognitive Systems,

University of Lübeck,

Lübeck, Germany

K. Kallenberg $\cdot$ J. Siemerkus

MR-Research in Neurology and Psychiatry,

Georg-August University of Göttingen,

Göttingen, Germany selected for this pilot study. Conventional MRI and fMRI were obtained. Transcranial magnetic stimulation was performed using a MagPro X100 stimulator and a standard figure-of-eight coil positioned by an Adept Viper s850 robot. The fMRI activation/Ri-TMS response pattern were compared. In two cases, Ri-TMS was additionally compared to intraoperative direct electrical cortical stimulation. Results Maximal MEP response of the $\mathrm{m}$. abductor digiti minimi was located in an area corresponding to the "hand knob" of the precentral gyrus for both hemispheres. Repeated Ri-TMS measurements showed a high reproducibility. Simultaneous registration of the MEP response for $\mathrm{m}$. brachioradialis, $\mathrm{m}$. abductor pollicis brevis, and $\mathrm{m}$. abductor digiti minimi demonstrated individual peak areas of maximal MEP response for the individual muscle groups. Ri-TMS mapping was compared to the corresponding fMRI studies. The areas of maximal MEP response localized within the "finger tapping" activated areas by fMRI in all six individuals.

Conclusions Ri-TMS is suitable for high resolution noninvasive preoperative somatotopic mapping of the motor cortex. Ri-TMS may help in the planning of neurosurgical procedures and may be directly used in navigation systems.

Keywords Functional MRI - Transcranial magnetic stimulation · Motor cortex · Brain mapping $\cdot$ Robotized neuronavigation $\cdot$ Electrical cortex stimulation

\section{Introduction}

The movements of the human body are controlled by the motor cortex located within the precentral gyrus, an anatomical structure readily identified on MRI [1] and by 
intraoperative mapping techniques during neurosurgical procedures [2]. The shape and exact location of the cortical motor areas varies among individuals and pathological conditions may cause distortion of functional motor areas and even shift of motor function to other regions [3]. However, the individual location of cortical motor areas is crucial for planning of neurosurgical procedures and preservation of motor function.

Direct electrical cortical stimulation (ECS) is well established and essential for localizing functional motor areas during neurosurgical procedures [2, 4]. For the planning of surgical approaches and procedures, preoperative functional maps of the motor cortex are required and in some cases the ability to precisely locate functional areas can become a determinant for feasibility of the neurosurgical intervention.

Blood-oxygen-level-dependent functional magnetic resonance imaging (BOLD-fMRI) is an established method in clinical neuroscience applied to visualize changes in magnetic field (in-)homogeneity caused by the oxygenation status of hemoglobin related to "brain activity" associated with specific tasks [5]. It can be used for mapping of the functional brain anatomy, which for motor- sensory- and speech areas have been widely used in neurosurgical decision-making [6]. The fMRI is the most widely used technique for non-invasive somatotopic mapping and is regarded as the gold standard today. The validity of this technique has been shown in several studies comparing fMRI with direct ECS [3, 7-9]. However, the spatial resolution of fMRI is limited because function is visualized indirectly by determination of changes in blood oxygen levels (BOLD), which are susceptible to alterations of the signal by large draining vessels or highly metabolically active tumors near the region of interest. Because changes in blood oxygen level require a larger number of active neurons, typically functional areas displayed by fMRI represent activity of relatively complex movements involving several muscle groups. The mapping of eloquent functional areas representing single muscles remains difficult.

Other techniques for non-invasive functional imaging include functional PET $[10,11]$, neuromagnetic recording [12], and conventional TMS [13]. To reduce interinvestigator variations and improve reproducibility, as well as the spatial accuracy, the latter has been assisted by stereotactical systems [14, 15] and frameless imageguidance navigation systems for positioning of TMS coils [16]. Denslow et al. compared an image-guided TMS technique to conventional hand-held function-guided coil placement and reported high precision and effectiveness of the image-guided TMS in reference to fMRI [17].

In this study, we investigate whether robot-assistance can be used as a feasible and safe technique for further improvement of image-guided transcranial magnet stimulation, and whether it allows the identification of cortical motor areas of individual muscles.

\section{Methods}

One healthy volunteer and five patients aged $53 \pm 11$ years (mean $\pm \mathrm{SD}$, three female) diagnosed with intracranial tumors (one meningeoma WHO grade I, one astrocytoma WHO grade II, one astrocytoma WHO grade III, and two glioblastomas WHO grade IV), neighboring the precentral gyrus but without apparent paresis at clinical examination were selected for this pilot study.

MRI-imaging, processing, and visualization

MRI studies were performed at $3 \mathrm{~T}$ (Magnetom Trio, Siemens Medical Solutions, Erlangen, Germany) using an eight-channel head coil. A T1-weighted whole-brain 3D-dataset (time to repetition $(\mathrm{TR}) /$ time to echo $(\mathrm{TE})=$ $11 / 4 \mathrm{~ms}$, flip angle $15^{\circ}, 1 \times 1 \times 1 \mathrm{~mm}^{3}$ isotropic resolution) and for fMRI-analysis a T2-weighted dataset (EPI, frequency-selective fat suppression, time to repetition TR/ time to echo $\mathrm{TE}=2,000 / 36 \mathrm{~ms}$, flip angle $70^{\circ}, 2 \times 2 \mathrm{~mm}^{2}$ resolution, $4 \mathrm{~mm}$ section thickness, 22 slices, top slice adjusted to the most superior proportion of the cortex; 129 acquisitions; total acquisition time $=4: 20 \mathrm{~min}$ ) were obtained.

The fMRI-paradigm consisted of $9 \times 18 \mathrm{~s}$ of resting state as control-condition alternating with $8 \times 12$ s of an active state starting with the control-condition. For the active state, subjects were instructed to successively touch the fingertips II-V with the thumb of either right or left hand as fast as possible (finger-tapping).

To avoid the T1 saturation effect the first two volumes of the T2-datasets were excluded from analysis. Preprocessing included mean intensity adjustment, slice scan time correction (cubic spline ascending interleaved), 3D motion correction (trilinear interpolation), and temporal filtering (high-pass/GLM-Fourier at 2 sines/cosines). The functional datasets were semi-automatically co-registered to the Talairach-transformed same subject T1-weighted wholebrain scan.

For the finger-tapping condition, a two-Gamma hemodynamic response function was used to calculate a general linear model (GLM).

For visualization, an appropriate statistical threshold for the GLM of each subject was defined. The positive suprathreshold voxels were overlayed as a color-coded map with higher $t$ values in blue and lower $t$ values in yellow on a semi-automatically calculated 3D-reconstruction of the cortex-white matter boundary of the $\mathrm{T} 1$-weighted dataset of the 
corresponding subject including all suprathreshold cortex voxels at a range of $3 \mathrm{~mm}$ to the cortex-white matter boundary.

\section{Robot-assisted image-guided TMS}

The system for robot-assisted image-guided TMS contains six main components (Fig. 1): A: TMS coil, B: robot, C: robot controller, D: personal computer, E: infrared tracking camera, and F: headband with reflective markers. Experiments were carried out using a standard figure-of-eight coil (Medtronic, MC-B70, Skovlunde, Denmark) attached to the articulated arm of an Adept Viper s850 robot (Adept Technology, Inc. Livermore, CA, USA), which was driven by a standard $\mathrm{PC}$ at $2.8 \mathrm{GHz}$ and software developed at the Institute for Robotics and Cognitive Systems, University of Lübeck, for image-guided robot-control [18]. A rubber headband carrying three passive Polaris reflective markers on a plastic frame was fixed to the head and tracked by a Polaris infrared stereo-optical tracking system (Northern Digital Inc., Waterloo, Ontario, Canada). The robot and the tracking system were registered to a common coordinate system using standard algorithms [19]. The coordinate system for the robot-guided TMS coil was defined by registration of three reference positions on the coil using a tracked pointer. This also determined the coordinate transformation from the robot end-effector (end of the robot arm) to the coil.
Registration of the spatial position of the cranium to the reconstruction of the cranial surface from MRI data was done by pointer acquisition of three standard landmarks (lateral orbital rims and tip of the nose) and up to 300 additional surface points [20].

For the transcranial magnetic stimulation, the coil target position was defined using the three-dimensional reconstruction of cranial MRI data. A stimulation target on the surface of the virtual head was selected and the orientation of the coil was calculated tangentially to cortical surface and $45^{\circ}$ to a saggital plane based on the reconstructed MRI data. The virtual coil position was then transformed to robot coordinates, which defined the movement of the robot to the corresponding target position relative to the patient's head. The position of the cranium was continuously tracked and the trajectory of the coil's path was adapted to movements of the head by a motion compensation module in real-time, which guaranteed a precise coil to target position and allowed free head movements during the study [21].

Transcranial magnetic stimulation was performed using a MagPro X100 MagOption stimulator (Medtronic, Dantec S.A., Skovlunde, Denmark) connected to a monitoring unit (Endeavor CR, Nicolet Biomedical, Dublin/Ohio, USA). The trigger signal for the stimulator and the recording unit of the Endeavor CR was generated by a script run on an external personal computer. The signal from stimulated muscles was registered by the Endeavor CR, band-pass-
Fig. 1 The upper panel shows the six main components of robot-guided TMS: $(A)$ TMS coil, $(B)$ robot, $(C)$ robot controller, $(D)$ computer, $(E)$ Polaris tracking system, $(F)$ headband. The lower left panel shows the examination set up and the RiTMS components $A, B, E, F$ and the MEP registration electrodes $(G)$. The right lower panel shows a surface reconstruction of a T1 MRI scan used for grid definition and robot control. The grid points stimulated by TMS are displayed as color-coded dots. The color indicates the magnitude of the MEP response evoked by Ri-TMS stimulation (red none, blue maximal)
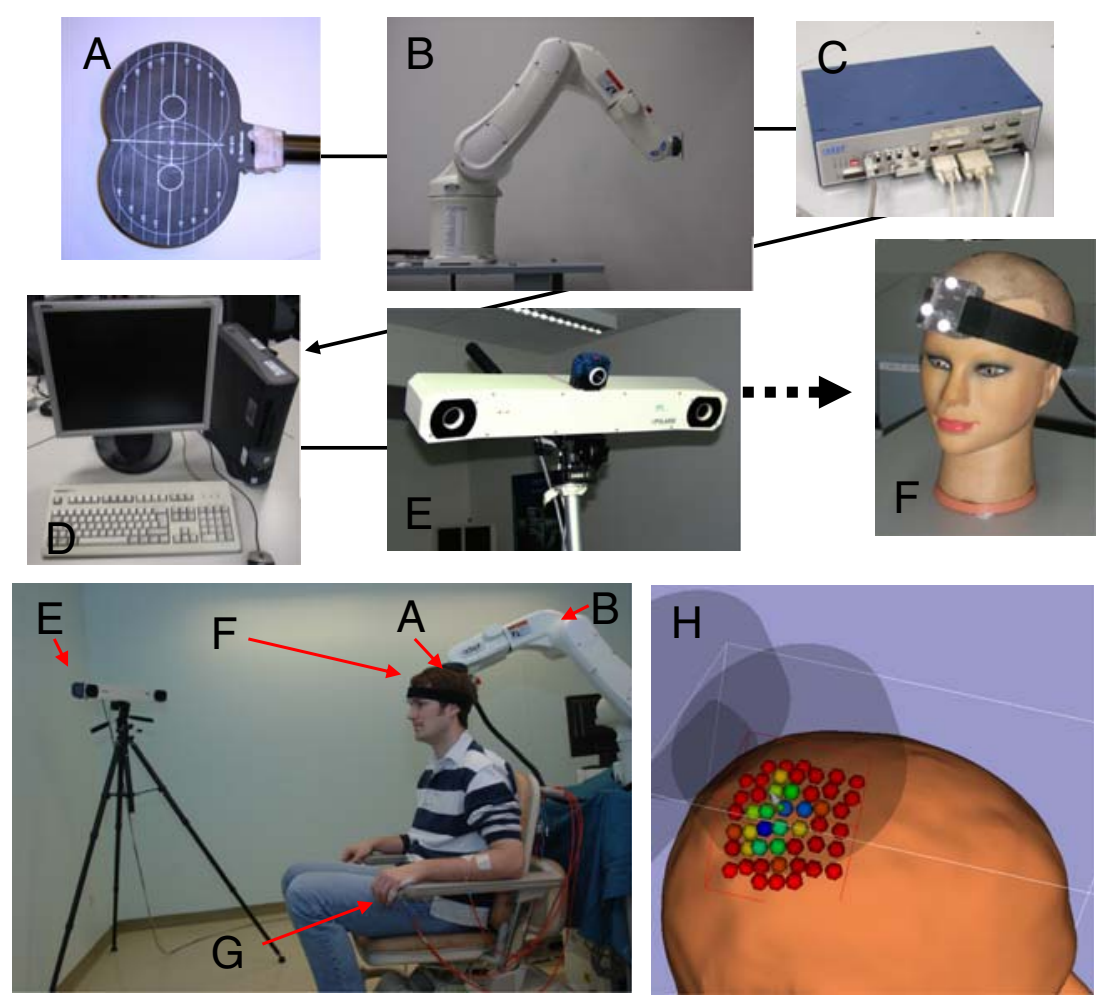
filtered between 2 and $2000 \mathrm{~Hz}$ and sampled at $5000 \mathrm{~Hz}$. The $\mathrm{m}$. brachioradialis, $\mathrm{m}$. abductor pollicis brevis, and $\mathrm{m}$. abductor digiti minimi were recorded using $\mathrm{Ag} / \mathrm{AgCl}$ bipolar surface electrodes. Both hemispheres were sequentially investigated by delivering ten biphasic pulses per target with a 5-s interstimulation interval $(0.2 \mathrm{~Hz})$. The analysis of the peak-to-peak amplitudes of the motor-evoked potentials (MEPs) was performed online using commercial software (Viasys, Nicolet Biomedical, Dublin/Ohio, USA). The final MEP response values used for construction of the motor map were calculated from the average of ten valid MEP response curves for each target. For selection of the initial stimulation intensity up to five targets corresponding to the position of the anatomically identified motor cortex (hand knob) were stimulated. The intensity was determined on the basis of reliable activation for at least one of the recorded muscles, peak-to-peak amplitude of the MEP $\geq 0.5 \mathrm{mV}$, without causing discomfort to the patients.

The distribution of the electric field of the TMS coil was determined in a previous experiment using the robot, a copper wire probe, and a PCS100 8-bit digital oscilloscope (Velleman Components N.V., Gavere, Belgium) with a sampling frequency of $800 \mathrm{kHz}$ [21]. The coil position data and the field characteristics of the coil allowed an approximation of the electric field distribution in the brain for each stimulation target. Assuming a functional relationship between the electric field strength at the anatomical representation of a muscle within the motor cortex and its MEP response, we calculated the most likely three-dimensional representation for the recorded muscle using an adapted version of the correlation ratio statistic (for details see [22]). The calculated field distributions were projected on the surface map of the brain, segmented from the diagnostic MRI.

\section{Procedure and evaluation}

For all patients and the healthy volunteer, conventional MRI and fMRI were obtained including protocol sequences for neuronavigation according to the manufactures instructions (VectorVision 2, BrainLAB, Feldkirchen, Germany) [23]. RiTMS was performed for both hemispheres. Maps of the brain surface were based on the segmented cortex structure from T1-weighted 3D MR sequence, acquired as described above. The fMRI and Ri-TMS data were projected on these maps, resulting in individual motor cortex maps.

The motor cortex maps generated by both techniques were analyzed by a neuroradiologist and a neurosurgeon. The location of the maximal activity/response was projected to the gyral pattern and compared relative to anatomical landmarks. The fMRI activation/Ri-TMS response pattern were scored in one of three categories: "Match" if the regions of fMRI activation and maximal RiTMS response located to the same anatomical site; "non- match" if the regions did not overlap; "match/nonmatch" for an partial overlap of areas of fMRI activation and RiTMS response.

Integration of Ri-TMS response into intraoperative neuronavigation

For patients scheduled for surgery, the regions of the maximal MEP response were transferred to the neuronavigation (VectorVision 2, BrainLAB, Feldkirchen, Germany). A direct transformation of maximal MEP response by TMS to intraoperative neuronavigation data was not possible because the navigation system used for RiTMS (Northern Digital Inc.) was based on a different coordinate system than the intraoperative neuronavigation (VectorVision 2). The data transformation was achieved by registration of scalp fiducial markers in both navigation systems regardless of the different registration protocols. This defined the marker positions in both coordinate systems $(\times 17)$. The regions of maximal MEP response were calculated by the divergence vectors from three different scalp fiducial markers. This mathematical operation allowed the location of the region of interest relatively to the fiducial markers and their position data of the intraoperative neuronavigation device.

\section{Intraoperative monitoring}

In three patients scheduled for surgery, standard neuronavigation-guided craniotomies were performed. Prior to and during the resection of the tumors, neuronavigationtracked ECS was carried out. For stimulation and registration of MEP response, an Endeavor CR neuromonitoring unit (Viasys, Nicolet Biomedical, Dublin/Ohio, USA) with a monopolar brain-stimulation electrode (Dr. Langer Medical GmbH, Waldkirch, Germany) was used. The regions of maximal response to cortical stimulation were directly compared to the calculated position of maximal MEP response by TMS.

\section{Results}

Patients and stimulation parameters

At the time of Ri-TMS, one of five patients required anticonvulsant medication because of a history of focal seizures. Medication of the other patients included dexamethasone and non-steroidal antiphlogistic therapy, which are not known to interfere with TMS. During the Ri-TMS, the patients were allowed to position and move their heads freely. Both hemispheres were examined, with the exception of one patient who developed a focal seizure and 
discontinued the mapping study. The stimulation intensity used for the mapping was selected between $40 \%$ and $45 \%$ $(43 \pm 2$, mean $\pm \mathrm{SD})$ of maximum stimulator output (MSO). This corresponds well with the intensities other authors applied for the same task [24]. Per hemisphere, an average of 38 grid points (range 32-60) was examined (Table 1). Measurements were started at a grid point directly above the anatomical hand knob identified on the surface reconstruction of MRI and continued in all planar directions at a grid width of $8 \mathrm{~mm}$ until no MEP response could be elicited in the muscle group of interest (Fig. 1, panel H).

\section{Cortical motor mapping}

The areas of maximal MEP response by Ri-TMS and fMRI activation were projected as maps on the surface reconstructions from MRI for each individual (Fig. 2). In a healthy volunteer, Ri-TMS was performed for both hemispheres. The maps of the calculated maximal MEP response demonstrated a maximal MEP response of the $\mathrm{m}$. abductor digiti minimi in an area corresponding to the "hand knob" of the precentral gyrus (Fig. 2a). The area of maximal MEP response of the $\mathrm{m}$. abductor digiti minimi was similar for the left and right hemispheres and corresponded well to the expected anatomical region (Fig. 2a and b). Repeated measurements using de novo registration and de novo definition of a grid system for Ri-TMS demonstrated a high reproducibility with the MEP response of the $\mathrm{m}$. abductor digiti minimi localizing to exactly the same anatomical site (Fig. 2a and c).
Simultaneous registration and discrimination of individual cortical motor areas

Simultaneous registration of the MEP response for $\mathrm{m}$. brachioradialis, $\mathrm{m}$. abductor pollicis brevis, and $\mathrm{m}$. abductor digiti minimi demonstrated individual peak areas of maximal MEP response for the individual muscle groups, which corresponded to their expected somatotopic representation within the precentral gyrus (Fig. 3 a, b and c). fMRI using a "finger tapping" paradigm showed activation of larger areas of the precentral gyrus and parts of the postcentral gyrus. The areas of maximal MEP response for $\mathrm{m}$. brachioradialis, $\mathrm{m}$. abductor pollicis brevis, and $\mathrm{m}$. abductor digiti minimi matched with the area of fMRI activation (Fig. 3d). In four of five patients with intracranial tumors near the motor cortex, Ri-TMS mapping of the effected hemisphere allowed identification of clearly distinct areas of maximal MEP response for the three muscles. In one patient (patient \#2) with a large postcentral histologically confirmed glioblastoma, Ri-TMS mapping localized the maximal MEP response for all three muscles to a relatively large area of the precentral gyrus. However, the areas of maximal MEP response for each of the three muscles overlapped, which did not allow discrimination of the individual peak areas of MEP response for the individual muscles. In a case of a low-grade glioma (patient \#5) involving the left central region and displacing the precentral gyrus rostrally, Ri-TMS mapping

Table 1 The table summarizes patient data, technical details, and results for all examined individuals examined by Ri-TMS (1 volunteer, 5 patients)

\begin{tabular}{|c|c|c|c|c|c|c|c|c|c|c|}
\hline & & \multirow[t]{2}{*}{ Diagnosis } & \multicolumn{5}{|c|}{ Ri-TMS } & \multicolumn{2}{|c|}{ fMRI/Ri-TMS } & \multirow{2}{*}{$\begin{array}{l}\text { IOP-ECS/Ri-TMS } \\
\text { Distance of peak areas } \\
\text { in } \mathrm{mm}\end{array}$} \\
\hline & & & $\begin{array}{l}\% \\
\mathrm{MSO}\end{array}$ & Grid & Time & $\begin{array}{l}\text { Right } \\
\text { hemisphere }\end{array}$ & $\begin{array}{l}\text { Left } \\
\text { hemisphere }\end{array}$ & $\begin{array}{l}\text { Right } \\
\text { hemisphere }\end{array}$ & $\begin{array}{l}\text { Left } \\
\text { hemisphere }\end{array}$ & \\
\hline Volunteer & $\begin{array}{l}\text { 45-year- } \\
\text { old male }\end{array}$ & No pathologies & 46 & 48 & 50 & $\begin{array}{l}\text { Complete } \\
\text { mapping }\end{array}$ & $\begin{array}{l}\text { Complete } \\
\text { mapping }\end{array}$ & Match & Match & No surgery performed \\
\hline $\begin{array}{l}\text { Patient \# } \\
1\end{array}$ & $\begin{array}{l}\text { 37-year- } \\
\text { old male }\end{array}$ & $\begin{array}{l}\text { Pre central } \\
\text { glioblastoma }\end{array}$ & 44 & 33 & 28 & $\begin{array}{l}\text { Complete } \\
\text { mapping }\end{array}$ & $\begin{array}{l}\text { Complete } \\
\text { mapping }\end{array}$ & Match & - & 26.3 after resection \\
\hline $\begin{array}{l}\text { Patient \# } \\
2\end{array}$ & $\begin{array}{l}\text { 58-year- } \\
\text { old female }\end{array}$ & $\begin{array}{l}\text { Post central } \\
\text { glioblastoma }\end{array}$ & 44 & 48 & 40 & $\begin{array}{l}\text { Incomplete } \\
\text { mapping }\end{array}$ & $\begin{array}{l}\text { Complete } \\
\text { mapping }\end{array}$ & $\begin{array}{l}\text { Match/ } \\
\text { nonmatch }\end{array}$ & - & $\begin{array}{l}\text { Not exposed during } \\
\text { surgery }\end{array}$ \\
\hline $\begin{array}{l}\text { Patient \# } \\
3\end{array}$ & $\begin{array}{l}\text { 68-year- } \\
\text { old female }\end{array}$ & $\begin{array}{l}\text { Meningeoma } \\
\text { central }\end{array}$ & 40 & 40 & 33 & $\begin{array}{l}\text { Complete } \\
\text { mapping }\end{array}$ & $\begin{array}{l}\text { Complete } \\
\text { mapping }\end{array}$ & Match & - & $\begin{array}{l}<5 \text { prior to resection, } \\
16.9 \text { after resection }\end{array}$ \\
\hline $\begin{array}{l}\text { Patient \# } \\
4\end{array}$ & $\begin{array}{l}\text { 49-year- } \\
\text { old female }\end{array}$ & $\begin{array}{l}\text { Prae central of } \\
\text { oligoastrocytoma }\end{array}$ & 42 & 36 & 30 & $\begin{array}{l}\text { Complete } \\
\text { mapping }\end{array}$ & $\begin{array}{l}\text { Complete } \\
\text { mapping }\end{array}$ & - & Match & No surgery performed \\
\hline $\begin{array}{l}\text { Patient \# } \\
5\end{array}$ & $\begin{array}{l}\text { 48-year- } \\
\text { old male }\end{array}$ & $\begin{array}{l}\text { Post central } \\
\text { astrocytoma }\end{array}$ & 44 & 32 & 27 & $\begin{array}{l}\text { Complete } \\
\text { mapping }\end{array}$ & $\begin{array}{l}\text { Incomplete } \\
\text { mapping }\end{array}$ & - & Match & No surgery performed \\
\hline
\end{tabular}

Technical details include: intensity of stimulation (\%MSO), number of grid points stimulated per hemisphere (grid), time required for mapping of one hemisphere (time). The results for the hemispheres affected by the tumor are shaded in gray. "Complete mapping" stands for a successful identification of distinct areas of maximal MEP response for $\mathrm{mm}$. brachioradialis, abductor pollicis brevis, and abductor digiti minimi within the precentral gyrus; while "incomplete mapping" describes an identification of areas of maximal MEP response with insufficient discrimination of the three muscle areas. If the areas of maximal MEP response to Ri-TMS are completely located within the area activated on fMRI, the result is rated as "match"; while "match/nonmatch" indicates an only partial overlap of fMRI and Ri-TMS 
Fig. 2 Projection of the MEP response on a segmented surface map of the brain (based on T1 MRI images). Red shows no excitability, blue shows the region of strongest MEP response. a Shows the area of maximal MEP response for the right $m$. abductor digiti minimi. b Shows the corresponding region for the left $\mathrm{m}$. abductor digiti minimi. c Shows a repeat examination illustrated for the area of maximal MEP response for the right $\mathrm{m}$. abductor digiti minimi (compare a) after 3 days using de novo registration and definition of a new grid system. Anatomical landmarks (central sulcus and hand knob) are highlighted in $\mathbf{d}$ for the right hemisphere

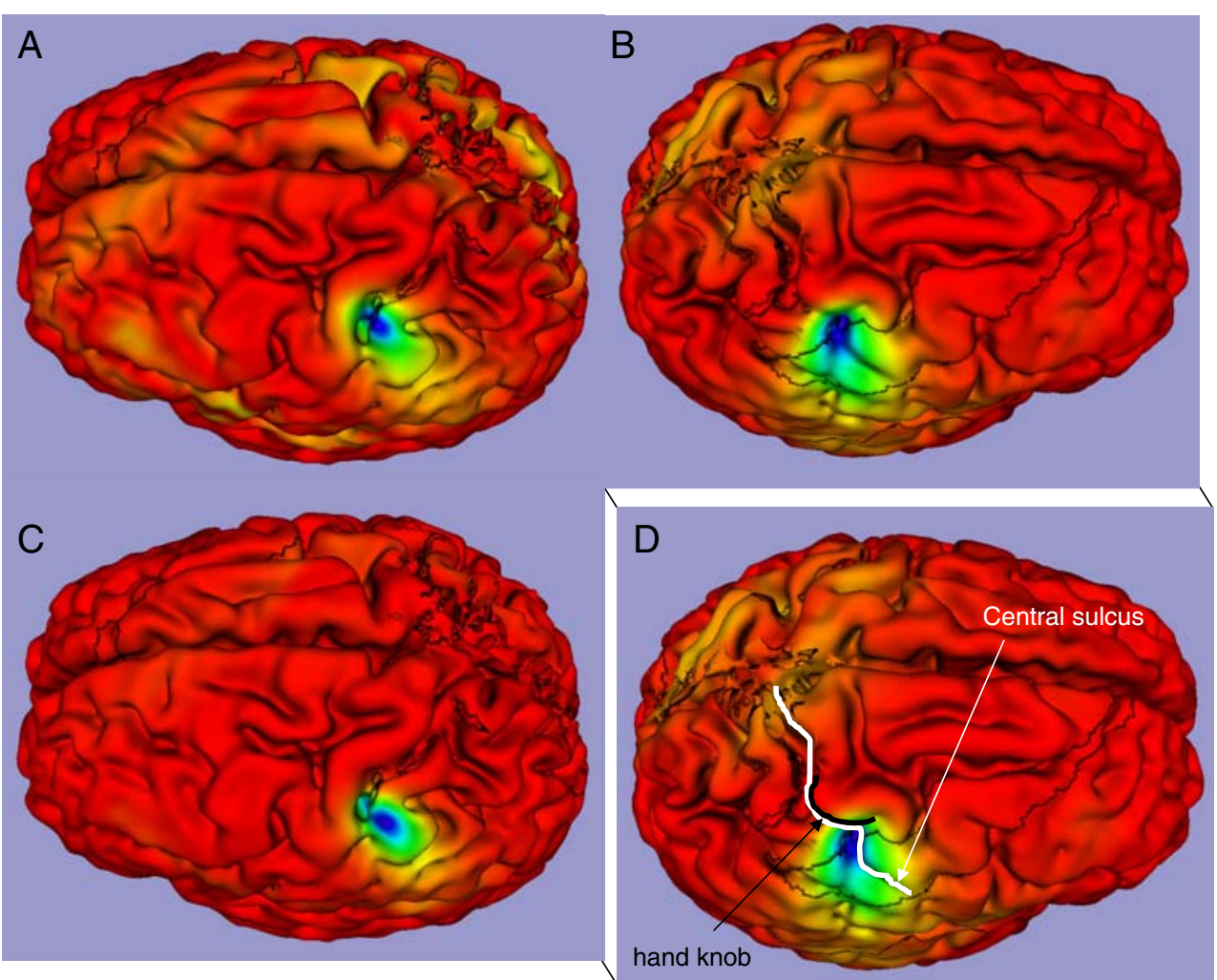

demonstrated highly circumscript areas of maximal MEP response for the $\mathrm{m}$. brachioradialis, $\mathrm{m}$. abductor pollicis brevis, and $\mathrm{m}$. abductor digiti minimi, but the three peak areas localized to a small area of the distorted precentral gyrus (Fig. 4). In both of these cases, identification of distinct cortical motor areas for the three muscles was possible for the unaffected contralateral hemisphere (Table 1).
Fig. 3 Simultaneous registration of the maximal MEP response for $\mathrm{m}$. brachioradialis (a), m. abductor pollicis brevis (b), and $\mathrm{m}$. abductor digiti minimi (c) by Ri-TCM. Corresponding fMRI for a finger-tapping paradigm (d)

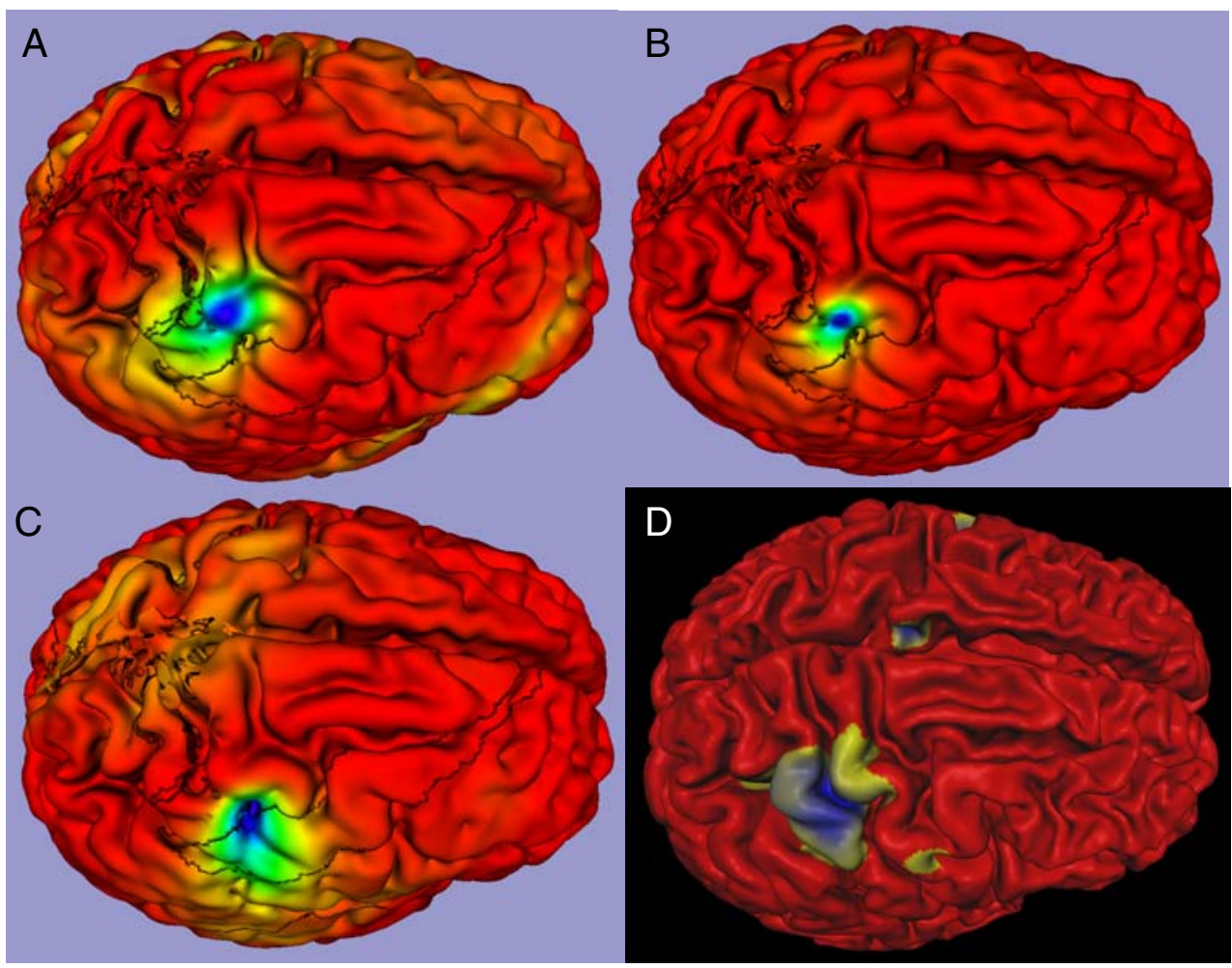




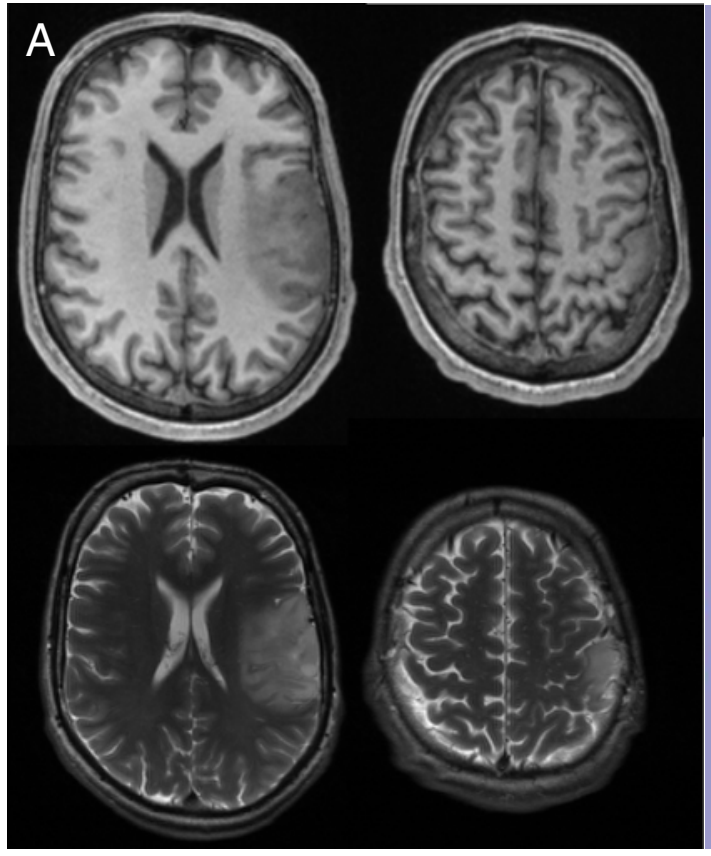

Fig. 4 Astrocytoma WHO grade II of the left central region (patient \#5). a T1- (upper panel) and T2- (lower panel) weighted MRI. Areas of maximal MEP response for $\mathrm{m}$. brachioradialis (b), $\mathrm{m}$. abductor

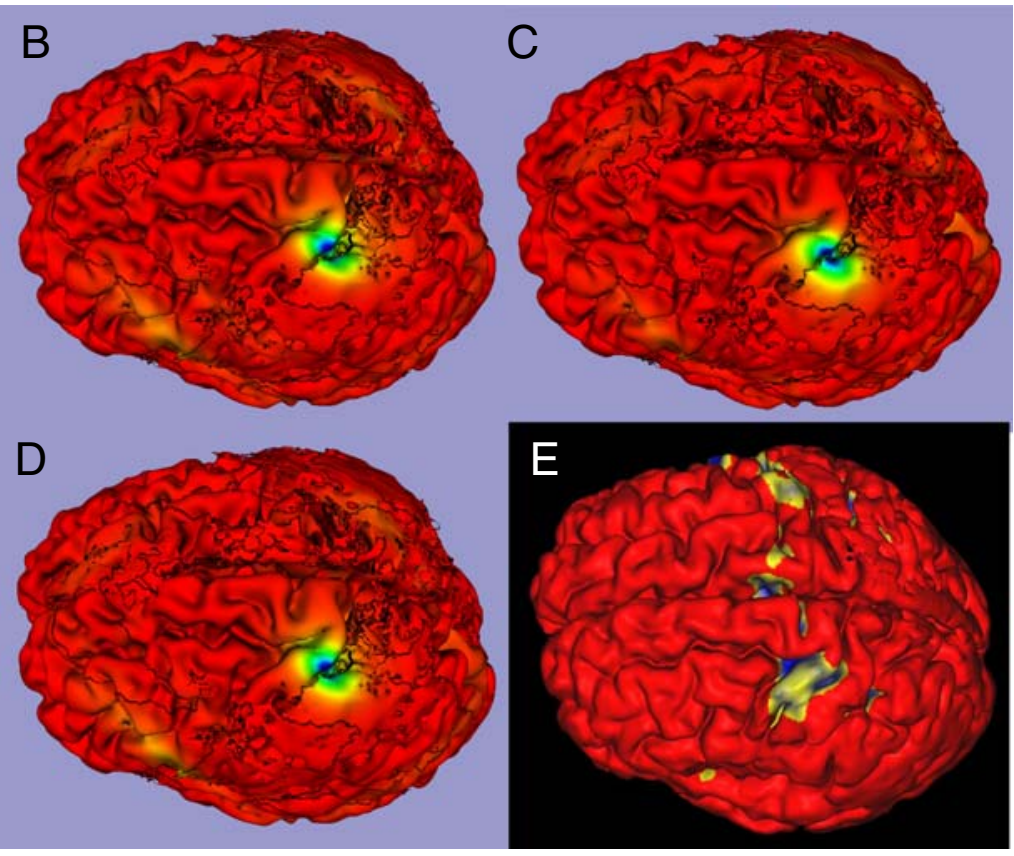

pollicis brevis (c), and $\mathrm{m}$. abductor digiti minimi (d) by Ri-TCM of the left hemisphere. $\mathbf{d}$ : Corresponding fMRI (finger-tapping paradigm)

maximal MEP response were registered using the neuronavigation (Fig. 5). Measurements of the cortical distance between the areas of peak MEP response to preoperative Ri-TCM and intraoperative ECS were performed. This analysis demonstrated distances between 5 and $26.3 \mathrm{~mm}$. For the latter case, access to the precentral gyrus could only be obtained after removal of a large tumor and significant volume shifts had occurred (compare Table 1.).

\section{Case illustration}

\section{Patient \#3}

A 68-year-old female presented with focal seizures of the left arm. MRI-imaging was done and a lesion with radiological characteristics of a small meningeoma immediately above the right central sulcus was diagnosed. An fMRI demonstrated that left-sided "finger tapping" resulted in activation within the motor cortex immediately adjacent, lateral, and anterior to the tumor (Fig. 5). Ri-TMS was performed and demonstrated clear delineation of individual areas of maximal MEP response for the $\mathrm{m}$. brachioradialis, $\mathrm{m}$. abductor pollicis brevis, and $\mathrm{m}$. abductor digiti minimi. The areas of maximum MEP response by Ri-TMS were transferred to the neuronavigation (Fig. 5b). A neuronavigation-guided craniotomy exposed the tumor and a small area of adjacent cortex which was examined by direct electrical cortex stimulation. The area of maximal MEP response by ECS for the muscles in hand and forearm in both patients. Areas of 


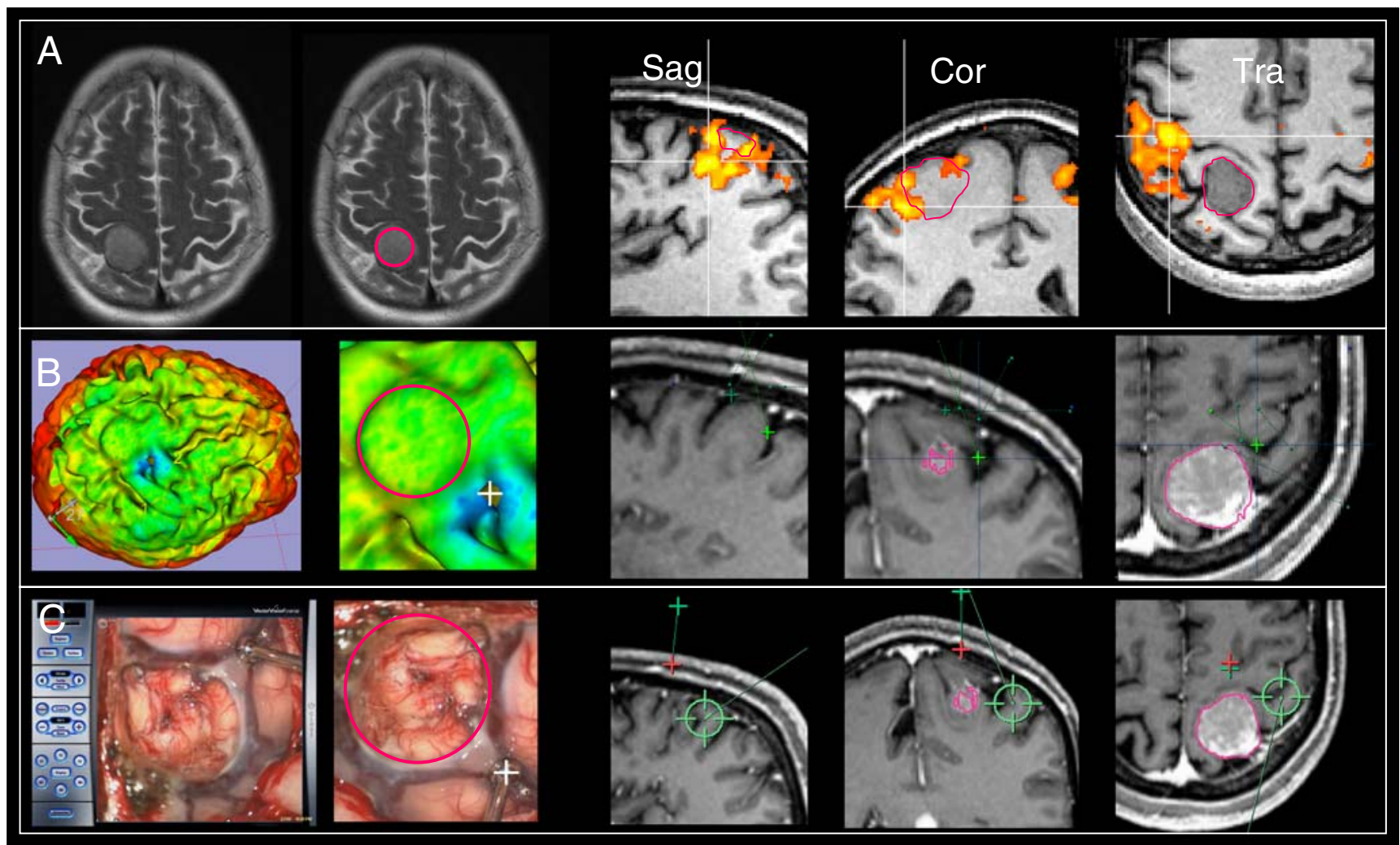

Fig. 5 Meningeoma above the right central sulcus causing focal seizures (patient \#3). a T2-weighted MRI and fMRI using a fingertapping paradigm. b Preoperative Ri-TCM (right) and integration of spatial Ri-TCM coordinates into the neuronavigation data set. c Intraoperative ECS mapping and comparison of data acquired for the maximal MEP response for ECS and Ri-TMS after tumor resection m. digiti was identified just lateral and anterior to the tumor. The cortical area was registered using the neuronavigation. Comparison of data acquired for the maximal MEP response for Ri-TMS and ECS showed that both areas localized to a morphologically distinct area of the proximal right "hand knob" within $5 \mathrm{~mm}$. However, due to the small craniotomy, more lateral aspects of the precental gyrus could only be exposed following resection of the tumor. Repeat cortical mapping by ECS now identified the cortical motor area of the $\mathrm{m}$. abductor pollicis brevis. Comparison of the spatial data of maximal MEP response by Ri-TCM and ECS now showed a mismatch of $16.9 \mathrm{~mm}$, possibly due to brain shift during the course of the operation as a consequence of loss of CSF and the resected tumor volume.

\section{Discussion}

TMS for somatotopic brain mapping

TMS is a reliable technique for somatotopic mapping of the motor cortex, which has been compared to fMRI imaging $[13,15,25-27]$ and direct ECS [14, 25]. The technique was much improved by introduction of image-guidance [17]. In this study, we investigated whether a further automatization by introduction of robot-assistance likewise results in a further improvement of the technique.

\section{Coil positioning and reproducibility}

Ri-TMS offers some advantages to conventional functionguided, stereotactic-, or image-guided-TMS. Robotized positioning of the coil was tightly controlled and defined by a constant grid width and a controlled position based on the surface reconstruction of the brain. It operated at a constant distance to the brain surface and the angulations of the coil was calculated relative to the individual shape of the brain surface. This minimizes inter-investigator variations. Repeated measurements on the same individual at a study interval of several days using de novo registration and planning resulted in identical results.

Spatial resolution of Ri-TMS

The cortical areas of maximal MEP response by Ri-TMS appeared to localize above the central sulcus but frequently involved areas within the pre- and postcentral gyrus, 
correlating very well with the fMRI findings in these patients. Accordingly, anatomical and physiological studies demonstrate that most motoneurons are located at the bottom of the central sulcus and the dorsal aspect of the precentral gyrus [28]. Although newer findings in primates suggest that also frontal non-primary motor areas were directly connected to the effector organs [29]. Whether this is true in humans has not yet been shown.

In one patient, where intraoperative exposure of the motor cortex allowed ECS prior to tumor resection, the mismatch was found to be less than $5 \mathrm{~mm}$. Lotze et al., in comparison, found in their study with five healthy volunteers, an average spatial difference between fMRI and TMS activation maxima of $13.9 \mathrm{~mm}$ [13] and Krings et al. found in an investigation of two patients with space-occupying tumors close to the central sulcus, more than $75 \%$ of the sites of maximal MEP response to TMS to be located within $1 \mathrm{~cm}$ of the sites of maximal ECS response [14].

Unfortunately, the size of our study (five patients and one volunteer) does not yet allow general assessment of maximum spatial resolution of Ri-TMS; however, the results of this pilot study already suggest a high accuracy.

Identification of cortical motor areas of individual muscle groups

Multi-channel registration of MEP allowed simultaneous analysis of the areas of maximal MEP for different muscle groups. Conceptually, this is not limited to three muscles (like in our study) and potentially allows generation of complex functional motor maps of a whole hemisphere by stimulation of a single grid. While this cannot be achieved by fMRI, as it visualizes activation of cortical areas induced by relatively complex tasks such as finger-tapping, involving groups of agonistic and antagonistic muscles; conventional image-guided TMS in combination with multi-channel MEP registration might allow discrimination of cortical motor areas for individual muscles as well. However, the anatomic differentiation of cortical motor areas is highly dependent to the spatial resolution of the technique. Therefore robotassistance might enhance discrimination by enhancing the spatial resolution. In our pilot study, differentiation and identification of cortical motor areas for individual muscle groups was achieved in all patients/probands who completed the Ri-TMS examination (at least in the non-affected hemisphere).

Investigation time and patient compliance

Besides technical issues like correct registration and coil placement, the accuracy of (image-guided) TMS is further limited by the ability of the patient to comply with the investigation. This is especially important for patients who suffer from severe comorbidities and/or neurological deficits which might restrict compliance. Dynamic robot positioning control allows the patient to move his head at will, and avoids the need for rigid fixation. This not only increases patient's comfort during the mapping procedure, but also increases safety by minimizing the risk of collision with the device by uncontrolled patient movements, for example, associated with seizures. Furthermore, it shortens investigation times significantly. The time required for RiTMS mapping largely depends on the number of grid points and the stimulation process itself, as coil positioning is extremely fast and no longer consumes significant time, even though the speed of the robot was limited to $3 \%$ of the maximal velocity $(228 \mathrm{~mm} / \mathrm{s})$ only.

Conceptually, after determination of the stimulation threshold, the mapping of a grid could be done automatically. In industrial settings, the type of robot used here operates at up to $7,600 \mathrm{~mm} / \mathrm{s}$ and maximal rotation: axis $1-$ $3250 \%$ s, axis $4-5375^{\circ} / \mathrm{s}$, and axis $6600 \%$ s.

\section{Outlook}

fMRI offers the possibility to localize non-motor functional areas, such as language [9, 30, 31] and sensory function [31]. Likewise, TMS can be used to localize these cortical areas by reversible suppression of neurological functions, such as cutaneous [32] and visual perception [33, 34], speech and counting [35], modulation and suppression of pain sensation [36], and therapeutic modulation of depression [37]. Some of these applications may benefit from application of Ri-TMS, especially in patients with restricted compliance. However, before Ri-TMS can be applied for these tasks, a large study has to be performed in order to exactly define the accuracy of Ri-TMS in comparison to ECS.

\section{Conclusions}

Ri-TMS is a feasible and safe technique for non-invasive somatotopic mapping of the motor cortex. The localization of functional areas within the motor cortex showed high intraindividual reproducibility and due to a high degree of automatization, was investigator-independent. Ri-TMS resolved the areas of maximal MEP response for individual muscles and therefore allowed identification of the cortical representation of motor control with high resolution. This suggests that the functional and spatial data acquired by RiTMS may help in the planning of neurosurgical procedures and may be directly used in navigation systems.

Acknowledgements T.F. was supported by an EC fellowship for Neuroscience Early Stage Research Training (NEUREST, MEST-CT2004-504193). 
Open Access This article is distributed under the terms of the Creative Commons Attribution Noncommercial License which permits any noncommercial use, distribution, and reproduction in any medium, provided the original author(s) and source are credited.

\section{References}

1. Missir O, Dutheil-Desclercs C, Meder JF, Musolino A, Fredy D (1989) Central sulcus patterns at MRI. J Neuroradiol 16(2):133-144

2. Penfield W, Boldry E (1937) Somatic motor and sensory representation in the cerebral cortex of man as studied by electrical stimulation. Brain 60:389-443

3. Lehéricy S, Duffau H, Cornu P, Capelle L, Pidoux B, Carpentier A, Auliac S, Clemenceau S, Sichez JP, Bitar A, Valery CA, Van Effenterre R, Faillot T, Srour A, Fohanno D, Philippon J, Le Bihan D, Marsault C (2000) Correspondence between functional magnetic resonance imaging somatotopy and individual brain anatomy of the central region: comparison with intraoperative stimulation in patients with brain tumors. J Neurosurg 92(4):589-598

4. Sala F, Lanteri P (2003) Brain surgery in motor areas: the invaluable assistance of intraoperative neurophysiological monitoring. J Neurosurg Sci 47(2):79-88

5. Frahm J, Bruhn H, Merboldt KD, Hänicke W (1992) Dynamic MR imaging of human brain oxygenation during rest and photic stimulation. J Magn Reson Imaging 2(5):501-505

6. Rao SM, Binder JR, Hammeke TA, Bandettini PA, Bobholz JA, Frost JA, Myklebust BM, Jacobson RD, Hyde JS (1995) Somatotopic mapping of the human primary motor cortex with functional magnetic resonance imaging. Neurology 45(5):919-924

7. Bizzi A, Blasi V, Falini A, Ferroli P, Cadioli M, Danesi U, Aquino D, Marras C, Caldiroli D, Broggi G (2008) Presurgical functional MR imaging of language and motor functions: validation with intraoperative electrocortical mapping. Radiology 248(2):579-589

8. Fandino J, Kollias SS, Wieser HG, Valavanis A, Yonekawa Y (1999) Intraoperative validation of functional magnetic resonance imaging and cortical reorganization patterns in patients with brain tumors involving the primary motor cortex. J Neurosurg 91 (2):238-250

9. Mueller WM, Yetkin FZ, Hammeke TA, Morris GL 3rd, Swanson SJ, Reichert K, Cox R, Haughton VM (1996) Functional magnetic resonance imaging mapping of the motor cortex in patients with cerebral tumors. Neurosurgery 39(3):515-520

10. Grafton ST, Woods RP, Mazziotta JC, Phelps ME (1991) Somatotopic mapping of the primary motor cortex in humans: activation studies with cerebral blood flow and positron emission tomography. J Neurophysiol 66(3):735-743

11. Viñas FC, Zamorano L, Mueller RA, Jiang Z, Chugani H, Fuerst D, Muzik O, Mangner TJ, Diaz FG (1997) [15O]-water PET and intraoperative brain mapping: a comparison in the localization of eloquent cortex. Neurol Res 19(6):601-608

12. Cheyne D, Kristeva R, Deecke L (1991) Homuncular organization of human motor cortex as indicated by neuromagnetic recordings. Neurosci Lett 122(1):17-20

13. Lotze M, Kaethner RJ, Erb M, Cohen LG, Grodd W, Topka H (2003) Comparison of representational maps using functional magnetic resonance imaging and transcranial magnetic stimulation. Clin Neurophysiol 114(2):306-312

14. Krings T, Buchbinder BR, Butler WE, Chiappa KH, Jiang HJ, Rosen BR, Cosgrove GR (1997) Stereotactic transcranial magnetic stimulation: correlation with direct electrical cortical stimulation. Neurosurgery 41(6):1319-1325

15. Neggers SF, Langerak TR, Schutter DJ, Mandl RC, Ramsey NF, Lemmens PJ, Postma A (2004) A stereotactic method for image- guided transcranial magnetic stimulation validated with fMRI and motor-evoked potentials. Neuroimage 21(4):1805-1817

16. Sparing R, Buelte D, Meister IG, Paus T, Fink GR (2008) Transcranial magnetic stimulation and the challenge of coil placement: a comparison of conventional and stereotaxic neuronavigational strategies. Hum Brain Mapp 29(1):82-96

17. Denslow S, Bohning DE, Bohning PA, Lomarev MP, George MS (2005) An increased precision comparison of TMS-induced motor cortex BOLD fMRI response for image-guided versus functionguided coil placement. Cogn Behav Neurol 18(2):119-126

18. Matthäus L, Trillenberg P, Bodensteiner C, Giese A, Schweikard A (2006) Robotized TMS for motion compensated navigated brain stimulation. Presented at the 20th International Computer Assisted Radiology and Surgery Congress, Osaka, Japan, June 28-July 1.

19. Horn B (1987) Closed-form solution of absolute orientation using unit quaternions. J Opt Soc Am A 4(4):629-642

20. Noirhomme Q, Ferrant M, Vandermeeren Y, Olivier E, Macq B, Cuisenaire O (2004) Registration and real-time visualization of transcranial magnetic stimulation with 3-d MR images. IEEE T Biomed E 51(11):1994-2005

21. Matthäus L, Giese A, Wertheimer D, Schweikard A (2006) Planning and analyzing robotized TMS using virtual reality. Stud Health Technol Inform 119:373-378

22. Matthäus L, Trillenberg P, Fadini T, Finke M, Schweikard A (2008) Brain mapping with transcranial magnetic stimulation using a refined correlation ratio and Kendall's tau. Stat Med 27 (25):5252-5270

23. Gumprecht H, Widenka D, Lumenta C (1999) BrainLab vectorvision neuronavigation system: technology and clinical experiences in 131 cases. Neurosurgery 44(1):97-104

24. Kammer T, Beck S, Thielscher A, Laubis-Herrmann U, Topka H (2001) Motor thresholds in humans: a transcranial magnetic stimulation study comparing different pulse waveforms, current directions and stimulator types. Clin Neurophysiol 112(2):250-258

25. Krings T, Buchbinder BR, Butler WE, Chiappa KH, Jiang HJ, Cosgrove GR, Rosen BR (1997) Functional magnetic resonance imaging and transcranial magnetic stimulation: complementary approaches in the evaluation of cortical motor function. Neurology 48(5):1406-1416

26. Macdonell RA, Jackson GD, Curatolo JM, Abbott DF, Berkovic SF, Carey LM, Syngeniotin A, Fabinyi GC, Scheffer IE (1999) Motor cortex localization using functional MRI and transcranial magnetic stimulation. Neurology 53(7):1462-1467

27. Niyazov DM, Butler AJ, Kadah YM, Epstein CM, Hu XP (2005) Functional magnetic resonance imaging and transcranial magnetic stimulation: effects of motor imagery, movement and coil orientation. Clin Neurophysiol 116(7):1601-1610

28. Rathelot JA, Strick PL (2006) Muscle representation in the macaque motor cortex: an anatomical perspective. Proc Natl Acad Sci USA 103(21):8257-8262

29. Teitti S, Määttä S, Säisänen L, Könönen M, Vanninen R, Hannula H, Mervaala E, Karhu J (2008) Non-primary motor areas in the human frontal lobe are connected directly to hand muscles. Neuroimage 40(3):1243-1250

30. Epstein CM, Meador KJ, Loring DW, Wright RJ, Weissman JD, Sheppard S, Lah JJ, Puhalovich F, Gaitan L, Davey KR (1999) Localization and characterization of speech arrest during transcranial magnetic stimulation. Clin Neurophysiol 110(6):1073-1079

31. Roux FE, Boulanouar K, Lotterie JA, Mejdoubi M, LeSage JP, Berry I (2003) Language functional magnetic resonance imaging in preoperative assessment of language areas: correlation with direct cortical stimulation. Neurosurgery 52(6):1335-1345

32. Seyal M, Masuoka LK, Browne JK (1992) Suppression of cutaneous perception by magnetic pulse stimulation of the human brain. Electroencephalogr Clin Neurophysiol 85(6):397-401 
33. Amassian VE, Cracco RQ, Maccabee PJ, Cracco JB, Rudell A, Eberle L (1989) Suppression of visual perception by magnetic coil stimulation of human occipital cortex. Electroencephalogr Clin Neurophysiol 74(6):458-462

34. Corthout E, Hallett M, Cowey A (2003) Interference with vision by TMS over the occipital pole: a fourth period. NeuroReport 14 (4):651-565

35. Pascual-Leone A, Gates JR, Dhuna A (1991) Induction of speech arrest and counting errors with rapid-rate transcranial magnetic stimulation. Neurology 41(5):697-702
36. Borckardt JJ, Smith AR, Reeves ST, Weinstein M, Kozel FA, Nahas Z, Shelley N, Branham RK, Thomas KJ, George MS (2007) Fifteen minutes of left prefrontal repetitive transcranial magnetic stimulation acutely increases thermal pain thresholds in healthy adults. Pain Res Manag 12(4):287-290

37. Dell'Osso B, Mundo E, D’Urso N, Pozzoli S, Buoli M, Ciabatti M, Rosanova M, Massimini M, Bellina V, Mariotti M, Carlo Altamura A (2009) Augmentative repetitive navigated transcranial magnetic stimulation (rTMS) in drug-resistant bipolar depression. Bipolar Disord 11(1):76-81 